\title{
Comparison between Accelerated Partial Breast Irradiation with multicatheter interstitial brachytherapy and Whole Breast Irradiation, in clinical practice
}

\author{
S. Garduño-Sánchez ${ }^{1,2} \mathbb{D} \cdot$ I. Villanego-Beltrán ${ }^{1,2} \cdot$ M. Dolores de las Peñas-Cabrera ${ }^{1,3} \cdot J^{\text {J Jaén-Olasolo }}{ }^{1,2} \mathbb{D}$
}

Received: 31 March 2021 / Accepted: 7 June 2021 / Published online: 2 July 2021

(c) Federación de Sociedades Españolas de Oncología (FESEO) 2021

\begin{abstract}
Purpose The aim of this study was to compare accelerated partial breast irradiation (APBI) with multicatheter interstitial brachytherapy (BT) and whole breast irradiation (WBI), in terms of toxicity, aesthetic result, quality of life and survival, in clinical practice.

Materials and methods A comparative study of two prospectively recorded cohorts of 76 breast cancer patients who complied with the recommendations of GEC-ESTRO for APBI was conducted. The main objective was toxicity, quality of life measured through validated questionnaires and the aesthetic results. Secondary objectives were overall survival and disease-free survival.

Results Seventy-six stage I/II breast cancer patients, with a mean age of 66 years entered the study. APBI group showed less acute $\mathrm{G} 1-2$ dermatitis $(51.4$ vs $94.9 \%, p<0.001)$ and late hyperpigmentation $(0$ vs $17.9 \%, p=0.04)$. There were no differences in aesthetic results, both assessed by the patient herself and by the doctor. Statistically significant differences in measures of quality of life were observed in favour of the APBI, both in EORTC QLQ-BR23 and body image scale questionnaires. With a median follow-up of 72 months (6 years), the estimated overall survival at 5 and 10 years was 96.8 and $77.7 \%$, respectively, and disease-free survival at 5 and 10 years was 91.1 and $69.4 \%$, respectively, without statistically significant differences between groups.

Discussion APBI is an attractive alternative in candidate patients with initial breast cancer, with benefits in acute toxicity and quality of life and fewer visits to the hospital, without compromising tumor control or survival.
\end{abstract}

Keywords Cancer $\cdot$ Breast $\cdot$ Brachytherapy $\cdot$ Accelerated partial breast irradiation

\section{Introduction}

Breast cancer is the most common malignant tumor worldwide, with more than 2 million new cases in 2020 and constitutes $25 \%$ of cancers in women [1]. Conservative surgery followed by radiotherapy (RT) has been the standard in the

S. Garduño-Sánchez

saragardu@gmail.com

1 Department of Radiation Oncology, Puerta del Mar University Hospital, Ana de Viya 21 Avenue, 11009 Cadiz, Spain

2 Biomedical Research and Innovation Institute of Cádiz (INiBICA) Research Unit, Puerta del Mar University Hospital, Cadiz, Spain

3 Department of Radiation Oncology, Rey Juan Carlos University Hospital, Gladiolo s/n, 28933 Madrid, Spain treatment of early stages for 3-4 decades, obtaining the same results as mastectomy $[2,3]$. However, the duration of radiotherapy, typically 5-6 weeks, makes it difficult to comply in regions with long distances to the hospital, especially in older and socially vulnerable women [4]. Subsequently, moderate hypofractionation regimens were imposed, lasting around 3 weeks, with the same results as conventional fractionation, as two large trials showed, one in Canada [5] and the other (START-B) in UK [6]. More recently, two studies in the UK have been carried out with five consecutive fractions (FAST-forward [7] and on a weekly basis (FAST) [8], also showing equivalent results.

On the other hand, numerous studies, both observational and comparative, show that about $90 \%$ of local recurrences occur in the immediate vicinity of the lumpectomy cavity. Based on this, new therapeutic strategies have been developed based on irradiation of a part of the breast, with the aim 
of reducing side effects with respect to WBI and several randomised clinical trials with this approach have been carried out. Occasionally, external RT has been used with similar fractionations to WBI $[9,10]$, although the most common is the use of "accelerated" fractionations (APBI), some with older technology (cobalt therapy, 2D-RT) [11] and others with more modern 3D-CRT $[12,13]$ and IMRT techniques [14]. Two large studies, one Italian (ELIOT) [15] and the other in the UK (TARGIT-A) [16], have carried out APBI with single-dose intraoperative RT (IORT), using electrons and $50 \mathrm{kV}$ energy X-ray, respectively. Other investigators have used both external RT and BT techniques in the APBI arm (Budapest and NSABP B-39/RTOG 0413 trials) [17, 18] and there is one trial (GEC-ESTRO) comparing APBI with multicatheter BT and WBI, both HDR and PDR [19].

From published clinical trials, at least five meta-analyses evaluating APBI versus WBI have been carried out. All show similar [20,21,22] or even better overall survival $[23,24]$ with APBI, in general with a better toxicity profile, although with conflicting results regarding local control.

Outside the scope of the operating room in IORT, BT allows, in addition to reducing the treatment time and number of fractions, a lower dose to the organs at risk, especially the skin, lungs, and heart. It is in this context that our study is placed, which aims to compare APBI with multicatheter interstitial BT vs WBI in clinical practice in terms of toxicity, aesthetic result, quality of life, local control, and survival.

\section{Materials and methods}

We conducted a comparative study of two contemporary prospectively recorded cohorts including a total of 76 patients, all women, who were treated with conservative surgery for early stage I-II breast cancer and who received adjuvant RT in our center, between 2008 and 2019. After $\mathrm{RT}$, patients were scheduled for physical examination at 6 weeks, every 6 months in the next 3 years and annually subsequently. Mammography was performed 6 months after radiotherapy and annually thereafter.

A cohort consisted of 33 consecutive patients treated with APBI using high dose rate (HDR) multicatheter interstitial BT with 192 Iridium sources and who met the GECESTRO recommendations for APBI [25]. Mainly older than 50 years, with infiltrating unicentric and unifocal tumors, in stages pT1-2 pN0-1mic M0, without extensive intraductal component or lymphovascular invasion, surgical margins at $\geq 2 \mathrm{~mm}$, and without prior chemotherapy. Patients who received APBI for second primary in the same breast after previous conservative surgery and external radiotherapy were excluded. The other cohort consisted of 40 patients randomly chosen from among those who received WBI using
3D-Conformal Radiotherapy (3D-CRT), in the same period of time and with the same inclusion criteria. The choice of one or the other treatment was due to different styles of practice among the professionals in our center.

The primary objectives of the study were (1) comparative analysis of toxicity, both acute and late, according to the CTCAE v4.0 (common terminology criteria for adverse events) criteria. For the evaluation of late effects, clinical criteria evaluated by physicians were also used, according to the presence or absence of certain signs (thickening of the breast and scar tissue, breast asymmetry, hyperpigmentation of the skin, and edema) and radiological, from posttreatment control mammograms, depending on the presence or absence of fibrosis, architectural distortion, retraction of breast tissue, and liponecrosis. (2) Measurement of quality of life, through self-administered questionnaires validated for breast cancer the modified QLQ-BR23 of the EORTC [26], composed of 22 items, excluding the one related to hair loss by chemotherapy, as none of the patients received that treatment. Individual analyses were carried out for each item and also aggregated by the following domains general physical, local physical, sexual, and psychological. (3) Measurement of body image, using a specific questionnaire Spanish version of the body image scale (S-BIS), made up of 10 items [27]. (4) Aesthetic result, using a visual analog scale (VAS) scored from 1 to 10 and evaluated, both by the patient herself and by the doctors.

As secondary endpoints, comparative survival analyses were performed between both cohorts. The overall and disease-free survival times were calculated from the date of surgery to the last date of follow-up or onset of the event: death or recurrence (locoregional and/or second tumor), respectively.

\section{Treatments}

The WBI group received 3D-CRT with six MV Rx on the entire mammary gland, using open and segmental tangential fields (field-in-field) and use of virtual wedges to homogenize the dose. The fractionation used was both conventional ( $2 \mathrm{~Gy} /$ Fx, s.i.d.), and in an START-B type hypofractionation regimen (2.67 Gy/Fx, s.i.d.). The indication for boost was established by your referring physician based on the center's protocol, taking into account prognostic factors of local recurrence, such as age, phenotype, tumor size, lymphovascular invasion, intraductal component, etc. Dosimetric data for planned treatment volume (PTV) were obtained from accumulated dosevolume histograms (DVH) volume percentage of PTV that received at least $95 \%$ of the prescribed dose (V95Gy) and percentage dose received for at least 98,2 , and $50 \%$ of the volume (D98, D2, and D50 respectively). Likewise, for the dosimetric parameters in organs at risk (ipsilateral lung and heart in left-sided breasts), mean doses, percentage of volume 
of the ipsilateral lung receiving a dose $\geq 20$ (conventional) and $\geq 17$ Gy (hypofractionation), and percentage of volume of the heart that received a dose $\geq 30$ (conventional) and $\geq 28$ Gy (hypofractionation).

All women in the APBI group received a 36 Gy fractionation into four b.i.d. fractions in 1 week, following the recommendations of the GEC-ESTRO [28, 29, 30]. In all cases, prior to implantation, an analysis of the available diagnostic images (mammography, ultrasound, MRI), an ultrasound study in the previous days to locate the seroma, and a CT scan with the templates placed in the foreseeable position were performed. With the integration of all these data, the implant was performed under sedation and with the help of perforated templates to guarantee a good geometric arrangement of the catheters. Dosimetry was performed by simulation CT with radio-opaque markers inside the comfort catheters (Elekta) and the Oncentra planning system. The dosimetric parameters were obtained from the DVH volume and V90\% of the PTV, CI (Conformity Index, referred to the PTV), volume of the implant that received $\geq 150$ and $200 \%$ of the prescribed dose (V150 and V200\% respectively), DHI (homogeneity index, referred to the implant), COIN (conformation index, referred to both, PTV and implant), and maximum dose in the skin.

\section{Statistical analysis}

Descriptive analyses were summarized by means with their standard deviation (SD) for continuous variables and by frequencies and proportions for categorical variables. The association analysis of categorical variables of more than two categories was performed using the Chi-square test $\left(\chi^{2}\right)$ and, for dichotomous variables, using Fisher's exact test. The comparison of means of continuous variables between the two cohorts was carried out using Student's $t$ test. To assess the concordance between the evaluation of the aesthetic result by patients and physicians, a reliability analysis was performed by calculating the Cronbach's alpha index. A correlation analysis was also performed using Pearson's coefficient.

The overall and disease-free survival curves were constructed according to the Kaplan-Meier method and the differences in survival between groups were compared using Mantel-Cox (log-rank) tests for censored data.

For all tests, the level of statistical significance was considered with $p$ values lower than $5 \%$, on both sides. For the statistical analysis, the SPSS statistical package, version 19.0 (SPSS Inc., Chicago, IL, USA) was used.

\section{Results}

\section{Characteristics of the study population}

Seventy-six women, with a mean age of 66 years, entered the study 39 with 3D-CRT WBI and 37 with APBI using high dose rate multicatheter BT. The median follow-up was 72 months (6 years), with no significant differences between groups.

The baseline characteristics of the patients are summarized in Table 1. As reported, no significant differences were found between groups for most of the baseline characteristics analysed laterality, location, histological type, degree of differentiation, phenotype, tumor size, status axillary, pathological stage, distance to the surgical margin, and type of surgery. In contrast, the patients in the APBI group were significantly older than those in the WBI (68.1 vs 63.5 years, $p=0.01$ ).

Table 2 shows some characteristics and dosimetric parameters of the applied treatments, both in the APBI and WBI groups. In WBl, the most used fractionation $(61.5 \%)$ was START-B type hypofractionation scheme ( $40.05 \mathrm{~Gy}$ in 15 s.i.d. and 3 weeks fractions), followed by conventional (50 Gy in 25 s.i.d. and 5 weeks fractions). 20 patients $(51.3 \%)$ received an additional dose (boost) on the lumpectomy cavity: 11 in the hypofractionation group and nine in the conventional group, until reaching total doses of 48-53 Gy and 60-66 Gy, respectively. The average V95\% of the PTV was $98.4 \%$. The mean pulmonary doses were 7.4 and 10.4 Gy for conventional and hypofractionated, respectively. The mean cardiac doses (only in left-sided tumors) were $4.6 \mathrm{~Gy}$ (conventional) and 4.2 (hypofractionated).

All patients in the APBI group received the same treatment regimen $36 \mathrm{~Gy}$ in four b.i.d. fractions. In most cases (94.6\%), three planes of vectors were used and the average number of catheters was 16 . The average volume of the PTV was $96.8 \mathrm{cc}$. On average, all the calculated indices (V90\%, CI, DHI and COIN) remained within the recommendations of the standard guidelines [30, 31]. The mean maximum dose received in the skin was $2.7 \mathrm{~Gy}$.

\section{Acute and late side effects}

$51.4 \%$ of the patients in the APBI group presented acute skin toxicity, all grade one of CTCAE v4, compared to $94.9 \%$ in the WBI group ( $82.1 \%$ grade one and $12.8 \%$ grade two). These differences were statistically significant $(p<0.001)$. No other early toxicity was seen. Regarding late toxicity, statistically significant differences were found in the percentage of hyperpigmentation in favour of APBI 
Table 1 Patient characteristics

\begin{tabular}{|c|c|c|c|c|c|c|c|}
\hline & \multicolumn{2}{|c|}{ Total $(n=76)$} & \multicolumn{2}{|c|}{ WBI $(n=39)$} & \multicolumn{2}{|c|}{ APBI $(n=37)$} & \multirow{2}{*}{$\begin{array}{l}\text { Dif } \\
p \text { value }\end{array}$} \\
\hline & $N$ & $\%$ & $n$ & $\%$ & $n$ & $\%$ & \\
\hline Age mean (SD) & $65.7(7.6)$ & & $63.5(7.0)$ & & $68.1(7.6)$ & & 0.01 \\
\hline Side & & & & & & & 0.82 \\
\hline Right & 45 & 59.2 & 24 & 61.5 & 21 & 56.8 & \\
\hline Left & 31 & 40.8 & 15 & 38.5 & 16 & 43.2 & \\
\hline Location & & & & & & & 0.81 \\
\hline Upper outer quadrant & 32 & 42.1 & 17 & 43.6 & 15 & 40.5 & \\
\hline Upper inner quadrant & 7 & 9.2 & 2 & 5.1 & 5 & 13.5 & \\
\hline Lower outer quadrant & 2 & 2.6 & 1 & 2.6 & 1 & 2.7 & \\
\hline Lower inner quadrant & 4 & 5.3 & 2 & 5.1 & 2 & 5.4 & \\
\hline Central & 1 & 1.3 & 0 & 0 & 1 & 2.7 & \\
\hline Overlapping & 30 & 39.5 & 17 & 43.6 & 13 & 35.1 & \\
\hline Histological type & & & & & & & 0.27 \\
\hline Invasive ductal & 69 & 90.8 & 35 & 89.7 & 34 & 91.9 & \\
\hline Invasive lobular & 2 & 2.6 & 2 & 5.1 & 0 & 0 & \\
\hline Ductal in situ & 1 & 1.3 & 1 & 2.6 & 3 & 8.1 & \\
\hline Other & 4 & 5.3 & 1 & 2.6 & 0 & 0 & \\
\hline Histologic grade & & & & & & & 0.07 \\
\hline G1 & 31 & 40.8 & 11 & 28.2 & 20 & 54.1 & \\
\hline $\mathrm{G} 2$ & 39 & 51.3 & 24 & 61.5 & 15 & 40.5 & \\
\hline G3 & 6 & 7.9 & 4 & 10.3 & 2 & 5.4 & \\
\hline Phenotype & & & & & & & 0.18 \\
\hline Luminal A & 58 & 76.3 & 27 & 69.2 & 31 & 83.8 & \\
\hline Luminal B & 18 & 23.7 & 12 & 30.8 & 6 & 16.2 & \\
\hline Tumor size $(\mathrm{mm})$ mean $(\mathrm{SD})$ & $13.1(6.3)$ & & $13.2(6.9)$ & & $12.9(5.6)$ & & 0.86 \\
\hline Axillary status & & & & & & & 0.67 \\
\hline pNO & 72 & 94.7 & 37 & 94.9 & 35 & 94.6 & \\
\hline pN1mic & 4 & 5.3 & 2 & 5.1 & 2 & 5.4 & \\
\hline Staging & & & & & & & 0.48 \\
\hline 0 (in situ) & 1 & 1.3 & 1 & 2.6 & 0 & 0 & \\
\hline I & 67 & 88.2 & 33 & 84.6 & 34 & 91.9 & \\
\hline II & 8 & 10.5 & 5 & 12.8 & 3 & 8.1 & \\
\hline Surgical margin $(\mathrm{mm})$ mean $(\mathrm{SD})$ & $7.2(4.1)$ & & $6.9(4.1)$ & & $7.6(4.1)$ & & 0.49 \\
\hline Type of surgery & & & & & & & 0.11 \\
\hline Lumpectomy + SLNB & 73 & 96.1 & 39 & 100 & 34 & 91.9 & \\
\hline Lumpectomy + ALND & 3 & 3.9 & 0 & 0 & 3 & 8.1 & \\
\hline
\end{tabular}

Statistically significant differences in bold

Dif differences between WBI and APBI, SD standard deviation, SLNB sentinel lymph-node biopsy, ALND axillary lymph-node dissection

( 0 vs $17.9 \%, p=0.04$ ), but not in thickening of skin, asymmetry, and edema.

Late radiographic findings showed a higher rate of architectural distortion $(83.3$ vs $34.2 \%, p<0.001)$ and skin retraction (44.8 vs $15.8 \%, p=0.01)$ in the APBI group.

\section{Aesthetic result and quality of life}

The assessment of the aesthetic result, by VAS with a score of $0-10$, reported by the patient herself and by her doctor, was 8.3 and 8.4, respectively. A high degree of concordance between both assessments was demonstrated, with a Cronbach's alpha index of 0.84 . Likewise, a statistically significant correlation was observed between both measures, with a Pearson correlation coefficient of $0.72(p<001)$. Table 3 shows the toxicity data, both acute and late.

Tables 4, 5 show the results obtained in 50 patients, 25 in each group, that were recruited to a patient-reported substudy assessed by the EORTC QLQ-BR23 qualityof-life questionnaire and the body image scale (S-BIS), 
Table 2 Treatment and dosimetric characteristics

\begin{tabular}{|c|c|c|}
\hline \multicolumn{3}{|l|}{ WBI (3D-CRT) $n=37$} \\
\hline \multicolumn{3}{|l|}{ Fractionation $n(\%)$} \\
\hline Conventional (50 Gy) & 6 & $15.4 \%$ \\
\hline Conventional + boost $(60-66 \mathrm{~Gy})$ & 9 & $23.1 \%$ \\
\hline HypoFx START-B (40 Gy) & 13 & $33.3 \%$ \\
\hline HypoFx START-B + boost (48-53 Gy) & 11 & $28.2 \%$ \\
\hline \multicolumn{3}{|l|}{ Dosimetric parameters mean (SD) } \\
\hline PTV volume & $989 \mathrm{cc}$ & 195 \\
\hline PTV V95\% & $98.4 \%$ & 1.6 \\
\hline PTV D98\% & $95.6 \%$ & 2.7 \\
\hline PTV D2\% & $104.4 \%$ & 1.6 \\
\hline PTV D50\% ${ }^{\mathrm{a}}$ & $100.9 \%$ & 3.7 \\
\hline \multicolumn{3}{|l|}{ Conventional } \\
\hline Ipsilateral lung mean dose & 7.4 Gy & 4.1 \\
\hline Ipsilateral lung V20Gy & $10.3 \%$ & 3.7 \\
\hline Heart mean dose (left side only) & $4.6 \mathrm{~Gy}$ & 3.6 \\
\hline Heart V30Gy (left side only) & $4.9 \%$ & 4.0 \\
\hline \multicolumn{3}{|l|}{ Hypofractionation } \\
\hline Ipsilateral lung mean dose & 10.4 Gy & 9.6 \\
\hline Ipsilateral lung V17Gy & $11.3 \%$ & 9.2 \\
\hline Heart mean dose (left side only) & $4.2 \mathrm{~Gy}$ & 1.3 \\
\hline Heart V28Gy (left side only) & $3.3 \%$ & 2.6 \\
\hline \multicolumn{3}{|l|}{ APBI (Brachytherapy): $n=39$} \\
\hline \multicolumn{3}{|l|}{ Fractionation $n(\%)$} \\
\hline $8 \times 4 \mathrm{~Gy} / 1 \mathrm{w}$ & 37 & $100 \%$ \\
\hline \multicolumn{3}{|l|}{ Treatment planes $n(\%)$} \\
\hline 2 planes & 2 & $5.4 \%$ \\
\hline 3 planes & 35 & $94.6 \%$ \\
\hline Catheters mean (range) & $15.6(9-21)$ & \\
\hline \multicolumn{3}{|l|}{ Dosimetric parameters mean (SD) } \\
\hline PTV volume & $96.8 \mathrm{cc}$ & 48.4 \\
\hline PTV V90\% & $90.2 \%$ & 9.4 \\
\hline CI & $88.2 \%$ & 5.7 \\
\hline Implant V150\% & $32.9 \mathrm{cc}$ & 19.1 \\
\hline Implant V200\% & $10.1 \mathrm{cc}$ & 4.6 \\
\hline DHI & 0.76 & 0.07 \\
\hline $\mathrm{COIN}$ & 0.65 & 0.09 \\
\hline Maximum cutaneous dose & $2.7 \mathrm{~Gy}$ & 0.81 \\
\hline
\end{tabular}

$3 D-C R T$ three-dimensional conformal radiotherapy, HypoFx hypofractionation, $S D$ standard deviation, $C I$ conformity index referred to PTV, DHI dose homogeneity index referred to implant, COIN conformal index referred to both PTV and implant

${ }^{a}$ Relative to maximum dose prescription

respectively. Statistically significant differences were observed, in favour of the APBI, both in the general physical domain (1.2 vs $1.5, p=0.02)$, as well as in the local physical $(1.4$ vs $1.9, p<0.01)$ and psychological $(1.4$ vs $2.1, p<0.01)$, not so in the domain of sexuality ( 1.3 vs $1.5, p=0.24)$. Analysed individually, all the items that showed significant differences were in favour of the APBI. For example: Have you had skin problems on or in the area of your affected breast (e.g., itchy, dry, flaky)?, Have you felt physically less attractive as a result of your disease or treatment?, Have you been feeling less feminine as a result of your disease or treatment?, Did you find it difficult to look at yourself naked?, Have you been dissatisfied with your body?, Are you worried about your health in the future?

The results obtained with the body image scale showed statistically significant differences in favour of the APBI (1.2 vs $1.9, p<0.01)$ in each of the 10 items that compound the questionnaire.

\section{Overall survival and disease-free survival}

During the study follow-up period, no patient had locoregional recurrence and three developed metastases, two in the WBI group and one in the APBI group. Eleven patients, six in the WBI group and five in the APBI group, had a second cancer in a place other than the treated breast: two lung cancers (both smokers one developed a small cell lung cancer at 5 years, contralateral to the breast, the other, an adenocarcinoma ipsilateral to the breast, 7 years later), two liver, one tongue, one pancreas, one colon, one haematological, one from the bladder, one melanoma, and the other in the contralateral breast, at 8 years of free interval and with a very different immunohistochemical profile. Estimated overall survival at 5 and 10 years was 96.8 and $77.7 \%$, respectively, and disease-free survival at 5 and 10 years was 91.1 and $69.4 \%$, respectively, the median not having been reached for either of the two. There were no statistically significant differences between groups, both in overall survival (log-rank $p=0.97)$ and disease-free $(\log$-rank $p=0.82)($ Fig. 1, 2).

\section{Discussion}

Solid data support the use of APBI outside of clinical trials and scientific societies, both American (ABS [32], ASTRO [33]) and European (ESTRO [25]), have published recommendations in this regard. In this study, we have carried out a comparison between APBI $(n=37)$ with interstitial multicatheter HDR BT and WBI $(n=39)$ in clinical practice. To our knowledge, there are three available randomised clinical trials comparing APBI and WBI that include a group treated with these techniques in the APBI arm GEC-ESTRO, NSABP B-39/RTOG 0413, and Budapest trials, with 88, 120 , and 128 patients treated in this manner, respectively [17], [19], [18]. Although the APBI is a fascinating radiotherapy technique [34], it is still little used probably in relation to the experience of the centers and the availability of technologies [35].

The GEC-ESTRO trial, a multicentric randomised phase III non-inferiority trial, carried out in Europe, with 1148 
Table 3 Acute and late results

\begin{tabular}{|c|c|c|c|c|c|c|c|}
\hline & \multicolumn{2}{|c|}{ Total $(n=76)$} & \multicolumn{2}{|c|}{ WBI $(n=39)$} & \multicolumn{2}{|c|}{$\operatorname{APBI}(n=37)$} & \multirow{2}{*}{$\begin{array}{l}\text { Dif } \\
p \text { value }\end{array}$} \\
\hline & $N$ & $\%$ & $n$ & $\%$ & $n$ & $\%$ & \\
\hline Acute cutaneous toxicity (CTCAE v4.0) & & & & & & & $<0.001$ \\
\hline G0 & 20 & 26.3 & 2 & 5.1 & 18 & 48.6 & \\
\hline G1 & 51 & 67.1 & 32 & 82.1 & 19 & 51.4 & \\
\hline G2 & 5 & 6.6 & 5 & 12.8 & 0 & 0 & \\
\hline \multicolumn{8}{|l|}{ Late clinical toxicity } \\
\hline Thickening of skin & $60 / 69$ & 87.0 & $32 / 39$ & 82.1 & $28 / 30$ & 93.3 & 0.28 \\
\hline Asymmetry & $16 / 66$ & 24.2 & $7 / 39$ & 17.9 & $9 / 27$ & 33.3 & 0.24 \\
\hline Hyperpigmentation & $7 / 66$ & 11.0 & $7 / 39$ & 17.9 & $0 / 27$ & 0 & 0.04 \\
\hline Edema & $3 / 65$ & 4.6 & $3 / 38$ & 7.9 & $0 / 27$ & 0 & 0.26 \\
\hline \multicolumn{8}{|l|}{ Late radiographic findings } \\
\hline Fibrosis & $56 / 65$ & 86.2 & $32 / 38$ & 84.2 & $24 / 27$ & 88.9 & 0.72 \\
\hline Architectural distortion & $38 / 68$ & 55.9 & $13 / 38$ & 34.2 & $25 / 30$ & 83.3 & $<0.001$ \\
\hline Retractions & $19 / 67$ & 28.4 & $6 / 38$ & 15.8 & $13 / 29$ & 44.8 & 0.01 \\
\hline Liponecrosis & $9 / 65$ & 13.8 & $5 / 38$ & 13.2 & $4 / 27$ & 14.8 & 0.85 \\
\hline \multicolumn{8}{|l|}{ Cosmetic results (VAS) } \\
\hline Patient-reported $(n=46)$ mean $(\mathrm{SD})$ & $8.3(2.2)$ & & $7.9(2.6)$ & & $8.7(1.7)$ & & 0.20 \\
\hline Professional-reported $(n=26)$ mean (SD) & $8.4(1.6)$ & & $7.9(2.0)$ & & $8.8(0.8)$ & & 0.18 \\
\hline
\end{tabular}

Statistically significant differences in bold

Dif. differences between WBI and APBI, CTCAE v4.0 common terminology criteria for adverse event, version 4.0, $S D$ standard deviation, $B I-R A D S$ breast imaging reporting and data system, vas visual analogic scale

patients randomised to either WBI or APBI using multicatheter (HDR or PDR) BT [25]. The inclusion criteria are well known. Mainly: $>40$ years, unifocal, without lymph or blood-vessel invasion or extensive intraductal component, clear surgical margins, pTis-2a pN0-1mi. Median followup was 6.6 years. The non-inferiority of APBI in terms of local control, 5 year disease-free survival and 5 year overall survival, was proved. In a sub-analysis of this trial focused in early toxicity, Ott et al. found statistically significant differences in the incidence of grades 1-2 early side effects for APBI and WBI for skin toxicity (86 vs $21 \%$, respectively) [35]. In another sub-analysis focused on late toxicity, Polgar et al. found significant differences in the 5 year cumulative risk of grade $2-3$ skin toxicity (6.9\% in APBI group vs $10.7 \%$ in WBI group) [36].

The NSABP B-39/RTOG 0413, a multicentric randomised phase III equivalence trial, done in USA, with 4132 evaluable patients, did not met the criteria for equivalence to WBI in controlling ipsilateral breast tumor recurrence, its primary end-point [18]. The median time of follow-up was 10.2 years. The risk of a recurrence-free interval event was significantly higher for APBI than WBI, but the absolute difference between 10 year recurrence-free estimates was small $(<1.6 \%)$. Distant disease-free interval, overall survival, and disease-free survival were not different for APBI vs WBI. The highest CTCAE grade $\geq 2$ toxicity reported was higher in the WBI group (66 vs 54\%). It is difficult to make comparisons with this study, for two main reasons first, because they established very broad inclusion criteria. For example, they included $38 \%$ of women under 50 years of age, $10 \%$ with invasive node-positive (up to3), and $8 \%$ with multifocal tumors. Second, the group treated with multicatheter HDR BT was very underrepresented, only $6 \%$ compared to $21 \%$ with single-entry BT and $73 \%$ with 3D-CRT.

The Budapest trial has recently published its long-term (20 year) results [17]. It is a of a phase III clinical trial comparing the survival and cosmetic results of APBI and WBI in 258 patients. In the APBI group $(n=128), 88$ patients were treated with HDR multicatheter BT and 40 patients with electron beams. Patients with low-risk breast cancer were included: > 40 years (after 2001), unifocal, non-lobular invasive breast carcinomas, G1-2, without extensive intraductal component, clear surgical margins, pT1 pN0-1mi. Median follow-up time was 17 years. The authors did not find significant difference in the 20 year disease-free survival and overall survival. Significantly more patients had excellent or good cosmetic result (Harvard scale), assessed by treating radiation oncologist, in the APBI group (79.2 vs 59.5\%). No significant differences in late skin or fibrosis were found.

Like other authors, we have found a lower acute skin toxicity in the APBI group (51 vs 95\%). Also, a significantly lower incidence of hyperpigmentation was observed in the APBI group (2.9 vs 7.5\%). We have found more architectural distortion and skin retraction in late 
Table 4 Quality-of-life questionnaire EORTC QLQ-BR23
Table 5 Body image scale (S-BIS)

\begin{tabular}{|c|c|c|c|c|c|}
\hline \multirow[t]{2}{*}{ EORTC QLQ-BR23 } & \multirow{2}{*}{$\begin{array}{l}\text { Total }(n=50) \\
\text { Mean }(\mathrm{SD})\end{array}$} & \multirow{2}{*}{$\begin{array}{l}\text { WBI }(n=25) \\
\text { Mean }(\mathrm{SD})\end{array}$} & \multirow{2}{*}{$\begin{array}{l}\text { APBI }(n=25) \\
\text { Mean }(S D)\end{array}$} & \multicolumn{2}{|c|}{ Differences } \\
\hline & & & & $p$ value & $95 \% \mathrm{CI})$ \\
\hline General physical domain & $1.4(0.4)$ & $1.5(0.5)$ & $1.2(0.3)$ & 0.02 & {$[0.05-0.54]$} \\
\hline Dry mouth & $1.3(0.6)$ & $1.4(0.7)$ & $1.2(0.5)$ & 0.22 & {$[-0.14-0.57]$} \\
\hline Different flavour & $1.2(0.5)$ & $1.4(0.7)$ & $1.0(0.2)$ & 0.04 & {$[0.03-0.64]$} \\
\hline Discomfort in eyes & $1.2(0.6)$ & $1.2(0.7)$ & $1.3(0.5)$ & 0.68 & {$[-0.42-0.27]$} \\
\hline Hair loss & $1.2(0.4)$ & $1.2(0.5)$ & $1.2(0.4)$ & 0.71 & {$[-0.21-0.30]$} \\
\hline Sensation disease & $1.8(1.2)$ & $2.3(1.2)$ & $1.4(1.0)$ & 0.15 & {$[0.16-1.46]$} \\
\hline Blush/heat & $1.6(0.9)$ & $1.8(1.0)$ & $1.3(0.7)$ & 0.05 & {$[0-1.03]$} \\
\hline Headaches & $1.3(0.7)$ & $1.4(0.9)$ & $1.2(0.5)$ & 0.32 & {$[-0.22-0.65]$} \\
\hline Local physical domain & $1.7(0.6)$ & $1.9(0.6)$ & $1.4(0.5)$ & $<0.01$ & {$[0.20-0.86]$} \\
\hline Arm/shoulder pain & $1.7(0.9)$ & $2.0(0.9)$ & $1.4(0.7)$ & $<0.01$ & {$[0.22-1.14]$} \\
\hline Swelling MS & $1.4(0.7)$ & $1.6(0.9)$ & $1.2(0.5)$ & 0.07 & {$[-0.03-0.80]$} \\
\hline Difficulty raising MS & $1.7(0.8)$ & $1.9(0.9)$ & $1.5(0.8)$ & 0.07 & {$[-0.04-0.91]$} \\
\hline Pain in the chest affected & $1.9(0.9)$ & $2.1(0.8)$ & $1.6(0.9)$ & 0.07 & {$[-0.04-0.93]$} \\
\hline Chest swelling & $1.6(0.9)$ & $1.8(1.0)$ & $1.4(0.8)$ & 0.13 & {$[-0.12-0.91]$} \\
\hline Breast sensitivity & $1.8(0.9)$ & $2.1(0.9)$ & $1.5(0.8)$ & 0.06 & {$[-0.01-0.97]$} \\
\hline Skin involvement & $1.6(0.8)$ & $2.0(0.9)$ & $1.2(0.4)$ & $<0.01$ & {$[0.48-1.30]$} \\
\hline Sexual domain & $1.4(0.4)$ & $1.5(0.7)$ & $1.3(0.5)$ & 0.24 & {$[-0.13-0.52]$} \\
\hline Interest in sex & $1.5(0.6)$ & $1.5(0.7)$ & $1.4(0.5)$ & 0.31 & {$[-0.18-0.54]$} \\
\hline Active sexual life & $1.5(0.7)$ & $1.6(0.8)$ & $1.3(0.6)$ & 0.18 & {$[-0.13-0.65]$} \\
\hline Enjoy sex & $1.3(0.5)$ & $1.4(0.6)$ & $1.2(0.4)$ & 0.39 & {$[-0.18-0.45]$} \\
\hline Psychological domain & $1.7(0.7)$ & $2.1(0.9)$ & $1.4(0.4)$ & $<0.01$ & {$[0.31-1.09]$} \\
\hline Less attractive & $1.6(0.9)$ & $2.0(1.1)$ & $1.2(0.5)$ & $<0.01$ & {$[0.26-1.27]$} \\
\hline Less feminine & $1.5(0.9)$ & $1.7(1.1)$ & $1.2(0.6)$ & 0.05 & {$[0.00-1.02]$} \\
\hline Difficulty seeing your nudity & $1.5(1.0)$ & $1.8(1.2)$ & $1.2(0.6)$ & 0.03 & {$[-0.05-1.14]$} \\
\hline Body disillusion & $1.4(0.8)$ & $1.8(1.0)$ & $1.1(0.3)$ & $<0.01$ & {$[0.18-1.08]$} \\
\hline Concern about the future & $2.6(1.1)$ & $3.1(1.1)$ & $2.1(1.0)$ & $<0.01$ & {$[0.42-1.59]$} \\
\hline
\end{tabular}

Statistically significant differences in bold

$S D$ standard deviation, $C I$ confidence interval

\begin{tabular}{lllll}
\hline & Total $(n=50)$ & WBI $(n=25)$ & APBI $(n=25)$ & Differences \\
\cline { 5 - 6 } & Mean (SD) & Mean (SD) & Mean (SD) & $p$ value (95\% CI) \\
\hline Body image scale & $1.4(0.4)$ & $1.8(0.9)$ & $1.2(0.3)$ & $<\mathbf{0 . 0 1}[\mathbf{0 . 1 9 - 1 . 0 0 ]}$ \\
1. Self-conscious & $1.8(0.9)$ & $2.1(0.9)$ & $1.4(0.7)$ & $<\mathbf{0 . 0 1}[\mathbf{0 . 2 5}-\mathbf{1 . 2 0}]$ \\
2. Less physically attractive & $1.5(0.9)$ & $1.9(1.1)$ & $1.2(0.5)$ & $<\mathbf{0 . 0 1}[\mathbf{0 . 2 1 - 1 . 2 2}]$ \\
3. Dissatisfied with appearance & $1.4(0.8)$ & $1.7(1.0)$ & $1.2(0.5)$ & $\mathbf{0 . 0 5}[\mathbf{0 . 2 0}-\mathbf{0 . 9 3}]$ \\
4. Less feminine & $1.3(0.8)$ & $1.6(1.0)$ & $1.0(0.2)$ & $\mathbf{0 . 0 2}[\mathbf{0 . 1 1}-\mathbf{0 . 9 8}]$ \\
5. Difficult to see self-naked & $1.4(0.8)$ & $1.7(1.0)$ & $1.1(0.3)$ & $\mathbf{0 . 0 2}[\mathbf{0 . 1 0}-\mathbf{0 . 9 9}]$ \\
6. Less sexually attractive & $1.4(0.8)$ & $1.7(1.0)$ & $1.1(0.3)$ & $<\mathbf{0 . 0 1}[\mathbf{0 . 1 9}-\mathbf{1 . 0 6}]$ \\
7. Avoid people & $1.4(0.8)$ & $1.7(1.0)$ & $1.1(0.3)$ & $\mathbf{0 . 0 2}[\mathbf{0 . 0 9}-\mathbf{1 . 0 0}]$ \\
8. Body less whole & $1.4(0.8)$ & $1.8(1.0)$ & $1.1(0.3)$ & $<\mathbf{0 . 0 1}[\mathbf{0 . 2 2}-\mathbf{1 . 1 2}]$ \\
9. Dissatisfied with body & $1.4(0.8)$ & $1.7(1.0)$ & $1.2(0.5)$ & $\mathbf{0 . 0 3}[\mathbf{0 . 0 7}-\mathbf{1 . 0 2}]$ \\
10. Dissatisfied with scar & $1.5(0.8)$ & $1.8(1.0)$ & $1.2(0.6)$ & $\mathbf{0 . 0 2}[\mathbf{0 . 1 2}-\mathbf{1 . 0 6}]$ \\
\hline
\end{tabular}

Statistically significant differences in bold

$S D$ standard deviation, $C I$ confidence interval 
Fig. 1 Overall survival curve by groups
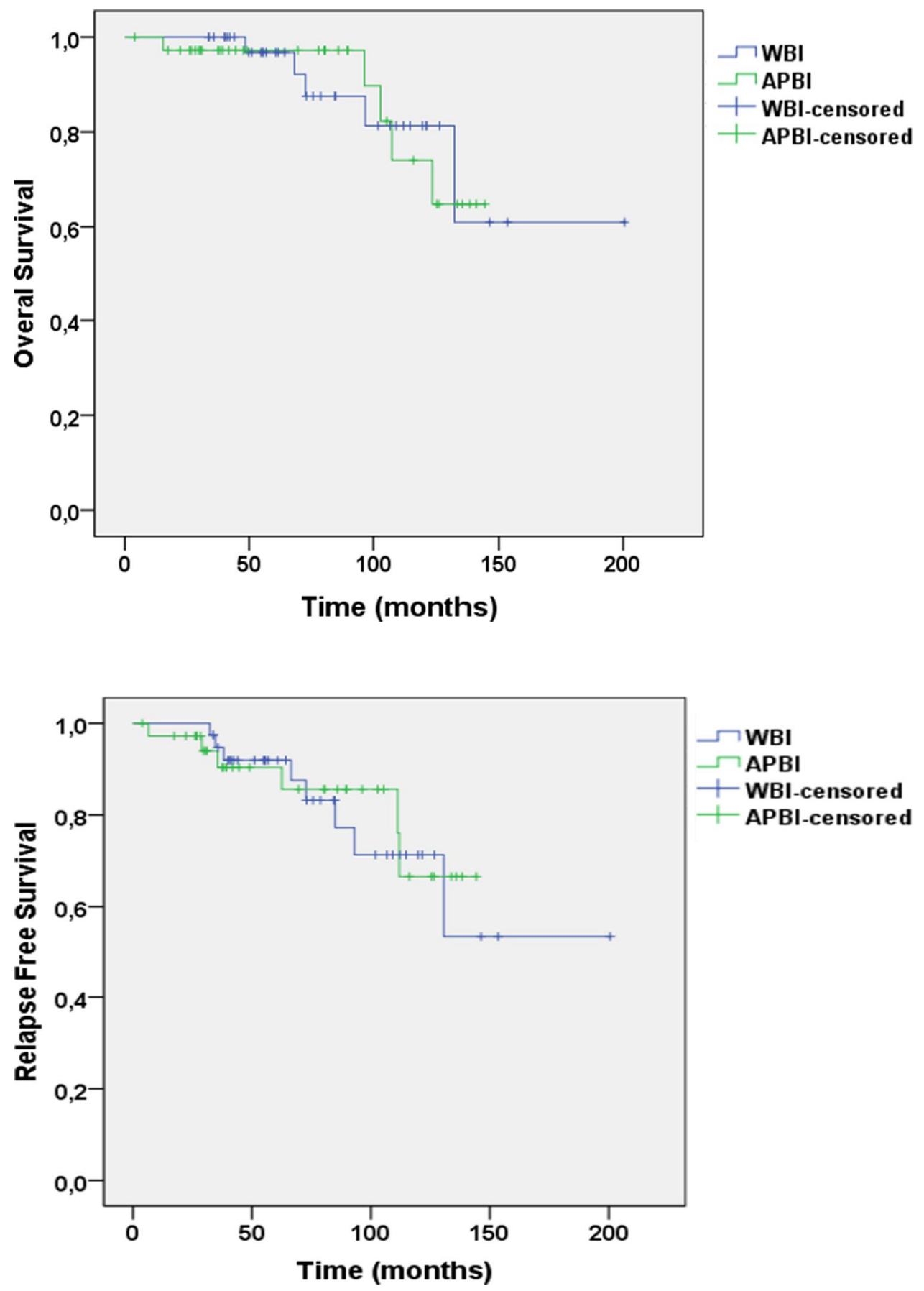

Fig. 2 Relapse free survival curve by groups radiological studies in the APBI group. However, as we will see later, these findings did not lead to a worse quality of life. Currently, IMRT-VMAT techniques are becoming the standard treatment in breast cancer. This implies fewer doses to OARS compared to 3D-CRT and probably less toxicity. In the present study, all WBI patients received 3D-CRT, which was our standard during the study period. Anyway, at least dosimetrically, there are still advantages for brachytherapy and, in any case, both techniques have not yet been compared in controlled clinical trials. The assessment of the aesthetic result by the doctor showed a high concordance with that carried out by the patient herself, without finding statistically significant differences between the two.

Quality of life, measured using validated questionnaires (EORTC QLQ-BR23 and S-BIS), was significantly better in the APBI group in both instruments. The researchers of the GEC-ESTRO trial also evaluated the quality of life of the patients, in their case using EORTC QLC-C30 questionnaire. They only found significant differences between 
groups in the breast symptoms scale, being worse for the WBI group.

Although there were no differences between groups regarding overall survival and disease-free survival, a longer follow-up period (median of 6 years in the present study) will help to obtain more robust quantitative data. All recurrences observed were in the form of metastasis or second primary in a different site than the treated breast. Two meta-analysis comparing APBI and WBI have shown better survival in the APBI group at 5 years [24] and at 10 years [23], but two others have shown worse local control in the APBI group [20, 21]. Other limitations of our study stem from the relatively small sample size and the retrospective nature of the study, with its inherent risk of selection bias, despite the WBI cohort being randomly selected. Another limitation arises from the heterogeneity of treatments in the WBI group, since hypofractionation began to replace the conventional one in our institution from 2010.

Starting in the 2010s, several clinical trials with external RT regimens in 5 days were published, resulting in comparable to more protracted regimens $[7,8]$. Based on this evidence, Spanish group of oncology radiotherapy (GEORM) strongly recommended a scheme based on these trials (REHMA) during the COVID-19 pandemic [37]. However, on the other hand, there are already recent phase II studies of APBI treatments with brachytherapy in 3 days and even with a single fraction. Future trials are likely to better clarify its role in clinical practice'.

In conclusion, APBI is an attractive alternative in candidate patients with initial breast cancer after breast conserving surgery, with benefits in acute toxicity and quality of life and fewer visits to the hospital, without compromising tumor control or survival. Apart from the fact of being an invasive procedure, multicatheter BT has dosimetric advantages compared to external RT, administering less doses to the skin, lung, and heart.

Author contribution All authors contributed equally to the study conception, design, material preparation, data collection, and analysis. All authors read and approved the final manuscript.

\section{Declarations}

Conflict of interest The author declared that they have no conflict of interest.

Ethical approval All the procedures performed were carried out in accordance with the institutional care protocols and with the Declaration of Helsinki of 1964 and its subsequent modifications or comparable ethical standards. The database used was retrospective withlack of patient intervention and all patient data were anonymized.

Informed consent Written inform consent for treatment was obtained from all individual participants included in the study.

\section{References}

1. Sung H, Ferlay J, Siegel RL, et al. Global cancer statistics 2020 GLOBOCAN estimates of incidence and mortality worldwide for 36 cancers in 185 countries. CA Cancer J Clin. 2021. https:// doi.org/10.3322/caac. 21660 .

2. Fisher B, Anderson S, Bryant J, et al. Twenty year follow-up of a randomized trial comparing total mastectomy, lumpectomy, and lumpectomy plus irradiation for the treatment of invasive breast cancer. N Engl J Med. 2002;347:1233-41. https://doi.org/ 10.1056/NEJMoa022152.

3. Veronesi U, Cascinelli N, Mariani L, et al. Twenty year followup of a randomized study comparing breast-conserving surgery with radical mastectomy for early breast cancer. N Engl J Med. 2002;347:1227-32. https://doi.org/10.1056/NEJMoa020989.

4. Pagano E, Di Cuonzo D, Bona C, et al. Accessibility as a major determinant of radiotherapy underutilization: a population based study. Health Policy. 2007;80:483-91. https://doi.org/ 10.1016/j.healthpol.2006.05.006.

5. Whelan TJ, Pignol J-P, Levine MN, et al. Long-term results of hypofractionated radiation therapy for breast cancer. N Engl J Med. 2010;362:513-20. https://doi.org/10.1056/NEJMoa0906 260.

6. Haviland JS, Owen JR, Dewar JA, et al. The UK standardisation of breast radiotherapy (START) trials of radiotherapy hypofractionation for treatment of early breast cancer: 10 year followup results of two randomised controlled trials. Lancet Oncol. 2013;14:1086-94. https://doi.org/10.1016/S1470-2045(13) 70386-3.

7. Brunt AM, Wheatley D, Yarnold J, et al. Acute skin toxicity associated with a 1-week schedule of whole breast radiotherapy compared with a standard 3-week regimen delivered in the UK FAST-Forward Trial. Radiother Oncol. 2016;120:114-8. https:// doi.org/10.1016/j.radonc.2016.02.027.

8. Brunt AM, Haviland JS, Sydenham M, et al. Ten year results of fast: a randomized controlled trial of 5-fraction wholebreast radiotherapy for early breast cancer. J Clin Oncol. 2020;38:3261-72. https://doi.org/10.1200/JCO.19.02750.

9. Dodwell DJ, Dyker K, Brown J, et al. A randomised study of whole-breast vs tumour-bed irradiation after local excision and axillary dissection for early breast cancer. Clin Oncol (R Coll Radiol). 2005;17:618-22. https://doi.org/10.1016/j.clon.2005. 07.018 .

10. Coles CE, Griffin CL, Kirby AM, et al. Partial-breast radiotherapy after breast conservation surgery for patients with early breast cancer (UK IMPORT LOW trial): 5 year results from a multicentre, randomised, controlled, phase 3, non-inferiority trial. Lancet. 2017;390:1048-60. https://doi.org/10.1016/ S0140-6736(17)31145-5.

11. Ribeiro GG, Magee B, Swindell R, et al. The Christie hospital breast conservation trial: An Update at 8 years from inception. Clin Oncol. 1993;5:278-83.

12. Rodríguez N, Sanz X, Dengra J, et al. Five year outcomes, cosmesis, and toxicity with 3-dimensional conformal external beam radiation therapy to deliver accelerated partial breast irradiation. Int J Radiat Oncol Biol Phys. 2013;87:1051-7. https://doi. org/10.1016/j.ijrobp.2013.08.046.

13. Olivotto IA, Whelan TJ, Parpia S, et al. Interim cosmetic and toxicity results from RAPID: a randomized trial of accelerated partial breast irradiation using three-dimensional conformal external beam radiation therapy. J Clin Oncol. 2013;31:403845. https://doi.org/10.1200/JCO.2013.50.5511.

14. Livi L, Meattini I, Marrazzo L, et al. Accelerated partial breast irradiation using intensity-modulated radiotherapy versus whole breast irradiation: 5 year survival analysis of a phase 
3 randomised controlled trial. Eur J Cancer. 2015;51:451-63. https://doi.org/10.1016/j.ejca.2014.12.013.

15. Veronesi U, Orecchia R, Maisonneuve P, et al. Intraoperative radiotherapy versus external radiotherapy for early breast cancer (ELIOT): a randomised controlled equivalence trial. Lancet Oncol. 2013;14:1269-77. https://doi.org/10.1016/S1470-2045(13) 70497-2.

16. Vaidya JS, Wenz F, Bulsara M, et al. Risk-adapted targeted intraoperative radiotherapy versus whole-breast radiotherapy for breast cancer: 5 year results for local control and overall survival from the TARGIT-A randomised trial. Lancet. 2014;383:603-13. https://doi.org/10.1016/S0140-6736(13)61950-9.

17. Polgár C, Major T, Takácsi-Nagy Z, et al. Breast-conserving surgery followed by partial or whole breast irradiation: twenty year results of a phase 3 clinical study. Int J Radiat Oncol Biol Phys. 2021;109:998-1006. https://doi.org/10.1016/j.ijrobp.2020.11.006.

18. Vicini FA, Cecchini RS, White JR, et al. Long-term primary results of accelerated partial breast irradiation after breast-conserving surgery for early-stage breast cancer: a randomised, phase 3, equivalence trial. Lancet. 2019;394:2155-64. https://doi.org/10. 1016/S0140-6736(19)32514-0.

19. Strnad V, Ott OJ, Hildebrandt G, et al. 5 year results of accelerated partial breast irradiation using sole interstitial multicatheter brachytherapy versus whole-breast irradiation with boost after breast-conserving surgery for low-risk invasive and in-situ carcinoma of the female breast: a randomised, phase 3, non-inferiority trial. Lancet. 2016;387:229-38. https://doi.org/10.1016/S01406736(15)00471-7.

20. Valachis A, Mauri D, Polyzos NP, et al. Partial breast irradiation or whole breast radiotherapy for early breast cancer: a meta-analysis of randomized controlled trials. Breast J. 2010;16:245-51. https://doi.org/10.1111/j.1524-4741.2010.00905.x.

21. Marta GN, Macedo CR, de Carvalho HA, et al. Accelerated partial irradiation for breast cancer: systematic review and meta-analysis of 8653 women in eight randomized trials. Radiother Oncol. 2015;114:42-9. https://doi.org/10.1016/j.radonc.2014.11.014.

22. Hickey BE, Lehman M, Francis DP, et al. Partial breast irradiation for early breast cancer. Cochrane Database Syst Rev. 2016. https:// doi.org/10.1002/14651858.CD007077.pub3.

23. Ye X-P, Bao S, Guo L-Y, et al. Accelerated partial breast irradiation for breast cancer: a meta-analysis. Transl Oncol. 2013;6:61927. https://doi.org/10.1593/tlo.13580.

24. Vaidya JS, Bulsara M, Wenz F, et al. Reduced mortality with partial-breast irradiation for early breast cancer: a meta-analysis of randomized trials. Int J Radiat Oncol Biol Phys. 2016;96:259-65. https://doi.org/10.1016/j.ijrobp.2016.05.008.

25. Polgár C, Van Limbergen E, Pötter R, et al. Patient selection for accelerated partial-breast irradiation (APBI) after breast-conserving surgery: recommendations of the Groupe Européen de Curiethérapie-European Society for Therapeutic Radiology and Oncology (GEC-ESTRO) breast cancer working group based on clinical evidence (2009). Radiother Oncol. 2010;94:264-73. https://doi.org/10.1016/j.radonc.2010.01.014.

26. Sprangers MA, Groenvold M, Arraras JI, et al. The European organization for research and treatment of cancer breast cancerspecific quality-of-life questionnaire module: first results from a three-country field study. J Clin Oncol. 1996;14:2756-68. https:// doi.org/10.1200/JCO.1996.14.10.2756.

27. Gómez-Campelo P, Bragado-Álvarez C, Hernández-Lloreda MJ, et al. The Spanish version of the body image scale (S-BIS): psychometric properties in a sample of breast and gynaecological cancer patients. Support Care Cancer. 2015;23:473-81. https:// doi.org/10.1007/s00520-014-2383-0.

28. Strnad V, Hannoun-Levi J-M, Guinot J-L, et al. Recommendations from GEC ESTRO breast cancer working group (I): target definition and target delineation for accelerated or boost partial breast irradiation using multicatheter interstitial brachytherapy after breast conserving closed cavity surgery. Radiother Oncol. 2015;115:342-8. https://doi.org/10.1016/j.radonc.2015.06.010.

29. Major T, Gutiérrez C, Guix B, et al. Recommendations from GEC ESTRO breast cancer working group (II): target definition and target delineation for accelerated or boost partial breast irradiation using multicatheter interstitial brachytherapy after breast conserving open cavity surgery. Radiother Oncol. 2016;118:199-204. https://doi.org/10.1016/j.radonc.2015.12.006.

30. Strnad V, Major T, Polgar C, et al. ESTRO-ACROP guideline: interstitial multi-catheter breast brachytherapy as accelerated partial breast irradiation alone or as boost - GEC-ESTRO breast cancer working group practical recommendations. Radiother Oncol. 2018;128:411-20. https://doi.org/10.1016/j.radonc.2018.04.009.

31. Shah C, Vicini F, Shaitelman SF, et al. The American brachytherapy society consensus statement for accelerated partial-breast irradiation. Brachytherapy. 2018;17:154-70. https://doi.org/10. 1016/j.brachy.2017.09.004.

32. Arthur DW, Vicini FA, Kuske RR, et al. Accelerated partial breast irradiation: an updated report from the American brachytherapy society. Brachytherapy. 2003;2:124-30. https://doi.org/10.1016/ S1538-4721(03)00107-7.

33. Smith BD, Arthur DW, Buchholz TA, et al. Accelerated partial breast irradiation consensus statement from the American society for radiation oncology (ASTRO). Int J Radiat Oncol Biol Phys. 2009;74:987-1001. https://doi.org/10.1016/j.ijrobp.2009.02.031.

34. Meduri B, Gregucci F, D'Angelo E, et al. Volume de-escalation in radiation therapy: state of the art and new perspectives. J Cancer Res Clin Oncol. 2020;146:909-24. https://doi.org/10.1007/ s00432-020-03152-7.

35. Ott OJ, Strnad V, Hildebrandt G, et al. GEC-ESTRO multicenter phase 3-trial: Accelerated partial breast irradiation with interstitial multicatheter brachytherapy versus external beam whole breast irradiation: Early toxicity and patient compliance. Radiother Oncol. 2016;120:119-23. https://doi.org/10.1016/j.radonc.2016. 06.019 .

36. Polgár C, Ott OJ, Hildebrandt G, et al. Late side-effects and cosmetic results of accelerated partial breast irradiation with interstitial brachytherapy versus whole-breast irradiation after breastconserving surgery for low-risk invasive and in-situ carcinoma of the female breast: 5 year results of a randomised, controlled, phase 3 trial. Lancet Oncol. 2017;18:259-68. https://doi.org/10. 1016/S1470-2045(17)30011-6.

37. Pardo R, Algara M, Montero-Fernández MA, et al. Diagnosis and locoregional treatment of patients with breast cancer during the COVID-19 pandemic. Revista de Senología y Patología Mamaria. 2020;33:61-7. https://doi.org/10.1016/j.senol.2020.04.002.

Publisher's Note Springer Nature remains neutral with regard to jurisdictional claims in published maps and institutional affiliations. 